CORRECTION

https://doi.org/10.1038/s41586-018-0099-x

\title{
Publisher Correction: Pervasive phosphorus limitation of tree species but not communities in tropical forests
}

Benjamin L. Turner, Tania Brenes-Arguedas \& Richard Condit

Correction to: Nature https://doi.org/10.1038/nature25789, published online 7 March 2018.

In this Letter, the $y$ axis of the right-hand panel of Fig. 2a was mislabelled 'Phosphomonoesterase' instead of 'Phosphodiesterase'. This error has been corrected online. 